\title{
Validity of Fusion Imaging of Hamster Heart obtained by Fluorescent and Phase-Contrast X-Ray CT with Synchrotron Radiation
}

\author{
J. Wu", T. Takeda*, Thet Thet Lwin", Q. Huo*, N. Sunaguchi" ${ }^{* *}$, T. Murakami**, \\ S. Mouri*, S. Nasukawa** T. Fukami* ${ }^{* *}$, T. Yuasa ${ }^{* *}$, \\ K. Hyodo ${ }^{* * *}$, H. Hontani ${ }^{* * * *}$, M. Minami ${ }^{*}$, and T. Akatsuka ${ }^{* *}$ \\ "Graduate School of Comprehensive Human Sciences, University of Tsukuba, Tsukuba, Ibaraki 305-8575 Japan, \\ ${ }_{* * *}^{* *}$ Faculty of Engineering, Yamagata University, Yonezawa, Yamagata 992-8510 Japan, \\ ${ }^{* * *}$ Institute of Material Science, High Energy Accelerator Research Organization, Tsukuba \\ Ibaraki 305-0801, Japan, \\ ***** Department of Computer Science and Engineering, Nagoya Institute of Technology, \\ Nagoya, Aichi 466-8555 Japan
}

\begin{abstract}
Fluorescent X-ray CT (FXCT) to depict functional information and phase-contrast X-ray CT (PCCT) to demonstrate morphological information are being developed to analyze the disease model of small animal. To understand the detailed pathological state, integration of both functional and morphological image is very useful. The feasibility of image fusion between FXCT and PCCT were examined by using ex-vivo hearts injected fatty acid metabolic agent $\left({ }^{127} \mathrm{I}\right.$ BMIPP) in normal and cardiomyopathic hamsters. Fusion images were reconstructed from each 3D image of FXCT and PCCT. ${ }^{127}$ I-BMIPP distribution within the heart was clearly demonstrated by FXCT with $0.25 \mathrm{~mm}$ spatial resolution. The detailed morphological image was obtained by PCCT at about $0.03 \mathrm{~mm}$ spatial resolution. Using image integration technique, metabolic abnormality of fatty acid in cardiomyopathic myocardium was easily recognized corresponding to anatomical structures. Our study suggests that image fusion provides important biomedical information even in FXCT and PCCT imaging.
\end{abstract}

Keywords: Fusion imaging, Fluorescent X-ray CT, Phase-contrast X-ray CT, Functional imaging, Molecular imaging, Cardiomyopathy, Small animal

PACS: 01.30.Cc, 07.85.Qe, 07.85.Nc, 32.50.+d, 32.30.Rj, 42.62.Be, 87.63.Lk, 87.19.Xx, 87.59.Fm

\section{INTRODUCTION}

Micro-imaging technique for small laboratory animal model of human diseases is an important tool for study on the cause, diagnosis and treatment of diseases. To obtain the biomedical information precisely from the morphological and/or functional point of view, imaging techniques with high-contrast and high-spatial resolution are required. Fluorescent X-ray CT (FXCT) and phase-contrast X-ray CT (PCCT) by using synchrotron radiation, which can reveal functional information and morphological structures, enable to obtain the fine image with high-contrast and high-spatial resolution because of their high physical sensitivity compared to conventional absorption-contrast technique [1-8]. However, it is difficult to confirm the detailed localization of abnormal area by FXCT only. Thus, fusion of functional FXCT image and anatomical PCCT image is quite useful for well understanding of the biomedical significance. Recently, we integrated the FXCT and PCCT images of the mouse brain and confirmed the validity of the fusion image to recognize the abnormal cerebral perfusion [9]. The aim of this study is to discuss the feasibility of fusion image for the evaluation the physiological state of hamster heart. 


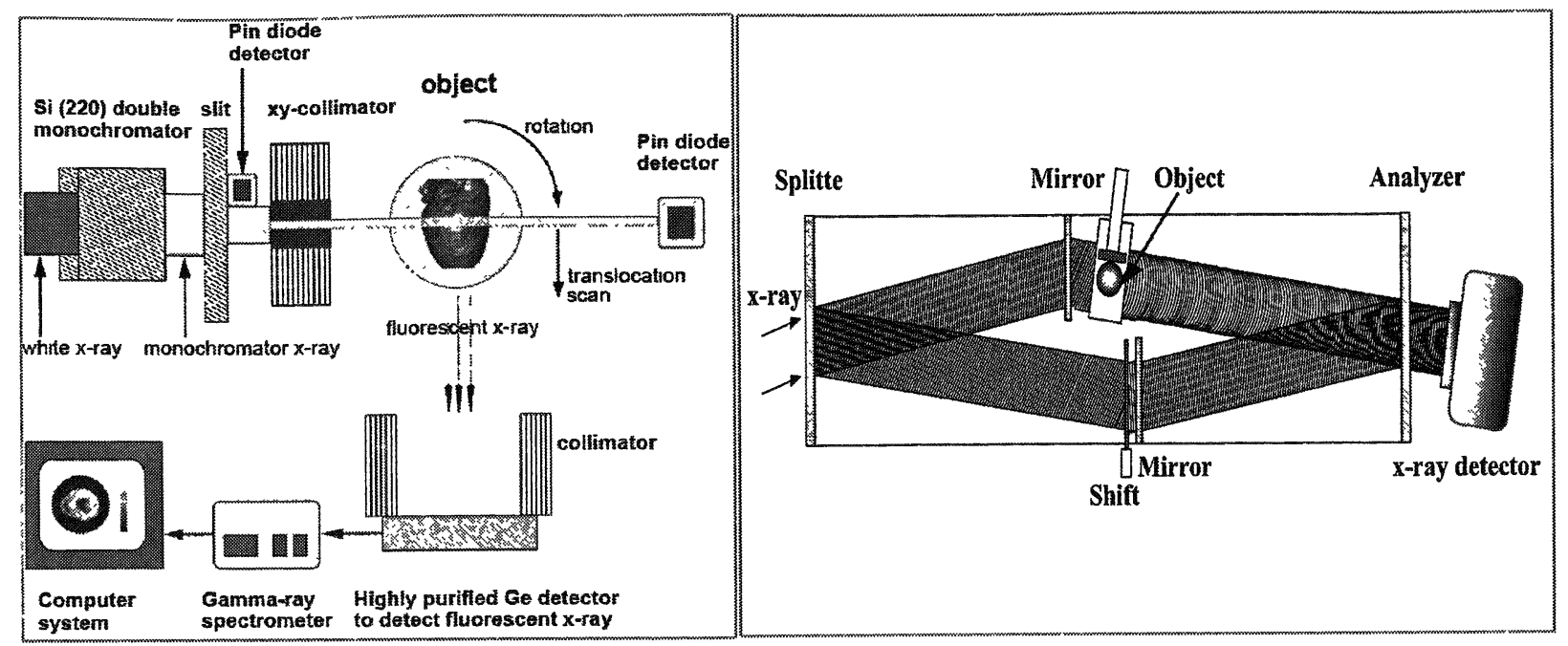

FIGURE 1. Schematic diagram of fluorescent (left) and phase-contrast (right) X-ray computed tomography system.

\section{METHODS AND MATERIAL}

\section{Animal Preparation}

Cardiomyopathic hamsters J2N-k and age matched normal J2N-n hamsters were used. Under anesthesia, the heart were extracted after 5-min intravenous injection of myocardial fatty acid metabolic agent, ${ }^{127} \mathrm{I}$ labeled $15-\mathrm{p}-$ (iodophenyl)-3-methlpentadecanoic acid ( ${ }^{127}$ I-BMIPP). Each heart was fixed by formalin for imaging. The experiment was approved by the Medical Committee for the Use of Animals in Research of the University of Tsukuba.

\section{Fluorescent X-ray CT Imaging}

FXCT system (Left half of Fig. 1) consists of a silicon (220) double crystal monochromator, an X-ray slit system, a scanning table, a fluorescent $\mathrm{X}$-ray detector, and two pin-diode for collecting incident and transmission X-ray data [5]. Fluorescent X-rays" were detected by a high purity germanium (HPGe, IGRET, EG\&G Ortec Ltd.) detector set placed perpendicular to the incident monochromatic X-ray beam. Monochromatic X-ray energy was set at $37 \mathrm{keV}$. X-ray beam was collimated to $0.25 \times 0.5 \mathrm{~mm}^{2}$ pencil beam, and object was scanned in 0.25 -mm translation step over the object region and $3^{\circ}$ rotation step over 180 degrees. Data acquisition time for each scanning step was $7 \mathrm{sec}$. The experiment was performed at the bending-magnet beam line BL-NE5A of Photon Factory, High Energy Accelerator Research Organization in Tsukuba, Japan.

\section{Phase-contrast X-ray CT Imaging}

Phase-contrast X-ray CT system (Right half of Fig. 1) consists of an asymmetrically cut silicon crystal, a monolithic X-ray interferometer, a phase shifter, an object cell and an X-ray CCD camera $[5,8]$. Fringe-scan technique was used and the X-ray energy was set at $35 \mathrm{keV}$ by the monochromator. The field of view was $24 \times 30$ $\mathrm{mm}^{2}$. The specimens were rotated inside the $20 \mathrm{~mm}$ thickness sample cells filled with formalin. Each phase map was calculated from 3 interference fringes by changing the phase of the reference beam, and each interference fringe was imaged at $5 \mathrm{~s}$ exposure and the number of projections was 250 over 180 degrees. The experiment was performed at a vertical wiggler beam line BL14C1 of the Photon Factory in Tsukuba, Japan. 


\section{Fusion Image Representation}

Three-dimensional (3D) images were reconstructed from the trans-axial image of FXCT and PCCT respectively. We determined heart surface contour from both three-dimensional images (3D-images) after resembling for describe with the same special resolution. Two images were fused interactively on displayed images by using 3D image manipulation software (Real INTAGE). Here, one is represented by gray scale image and the other was represented by pseudo-color for easy to manipulate them under operator's decision.

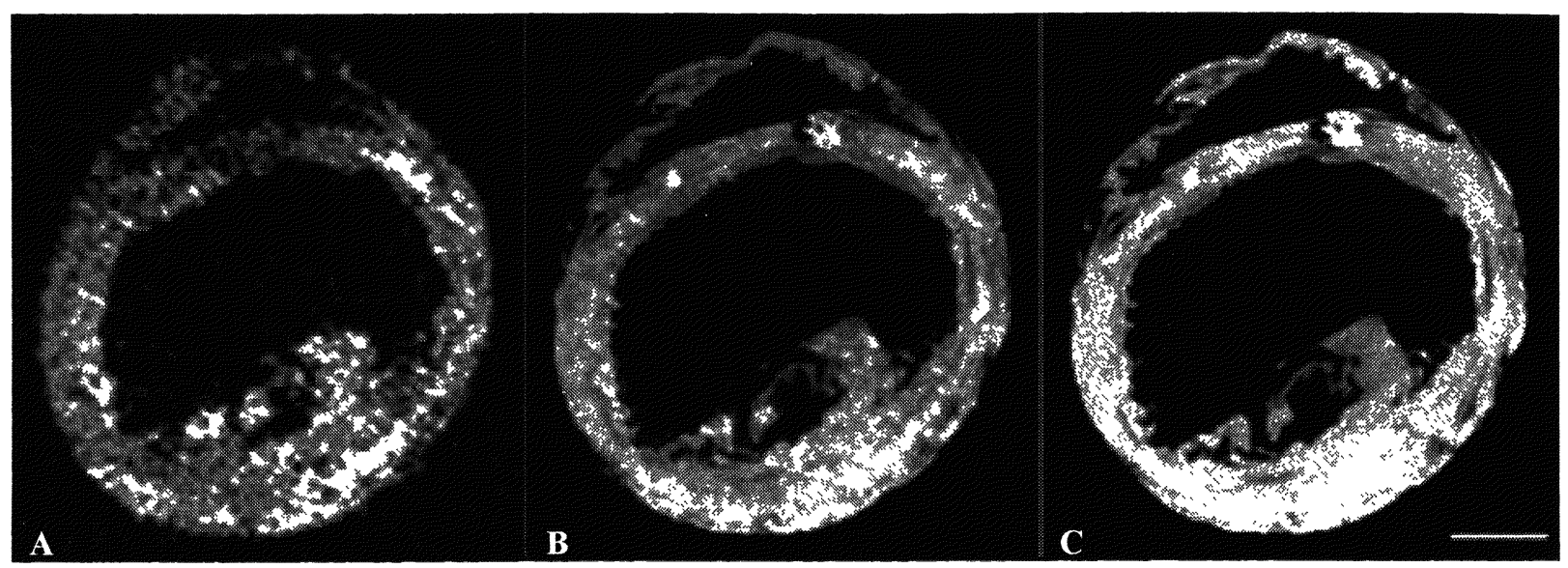

FIGURE 2. Images of a cardiomyopathic hamster obtained by fluorescent X-ray CT (FXCT) using ${ }^{127}$ I-BMIPP myocardial fatty acid metabolic agent (A), phase-contrast X-ray CT (PCCT) (C) and fused image of FXCT and PCCT (B). Patched low BMIPP uptake was observed clearly in left ventricular wall. The defect like area was demonstrated in septum which corresponded thrombus area on phase-contrast X-ray CT image. Scale Bar $=2 \mathrm{~mm}$.

\section{RESULTS AND DISCUSSION}

The extracted hearts of hamsters labeled ${ }^{127} \mathrm{I}-\mathrm{BMIPP}$ were imaged by FXCT and PCCT to obtain the functional and morphological images, respectively (Fig. $2 \mathrm{~A}$ and $\mathrm{C}$ ).

${ }^{127}$ I-BMIPP distribution within the left ventricular wall was clearly demonstrated by FXCT with $0.25 \mathrm{~mm}$ spatial resolution. Patched low uptake that corresponds to metabolic abnormality of the fatty acid, was observed in myocardium of J2N-k cardiomyopathic hamster (Fig. 2A), whereas normal hamster showed homogeneous distribution. These findings were the same as previously reported study with radioactive ${ }^{123}$ I-BMIPP [10]. However, detailed myocardial localization was difficult to recognize in FXCT image because the FXCT image demonstrates only metabolic condition in myocardium. On the other hand, PCCT with $0.03 \mathrm{~mm}$ spatial resolution, clearly depicted the morphological structures such as cardiac lumen, papillary muscle, and left and right ventricular wall (Fig. 2C). Then, we integrated the FXCT image and PCCT image for hamster heart. In fusion image, fatty acid metabolic abnormality in cardiomyopathic myocardium was easily recognized corresponding to detailed anatomical structures (Fig. 2B). This procedure is very useful to understand the biochemical state in myocardium.

Our results suggested that the image fusion was a quite significant technique to evaluate the exact and quantitative myocardial function of the small animal both in FXCT and PCCT with high spatial resolution. 


\section{ACKNOWLEDGMENTS}

We thank Akio Yoneyama $\mathrm{PhD}$, Xiaowei Chang $\mathrm{PhD}$ and Yoshinori Tsuchiya $\mathrm{PhD}$ for technical supports, Mr. Kouzou Kobayashi for his preparation of experimental apparatus, Nihon Medi-Physics Co., Ltd. Japan for supplying

${ }^{127}$ I-BMIPP, and Yukiko Kawata for help in preparing this article. This research was partially supported by Grant-InAid for Scientific Research (\#17591244, \#17390326, \#17659362), Special Coordination Funds and Grant-in-Aid for Scientific Research in Priority Areas (\#15070201) from the Ministry of Education, Culture, Sports, Science and Technology of the Japanese Government, and was performed under the auspices of High Energy Accelerator Research Organization (2005G308, 2005S2-001).

\section{REFERENCES}

1. T. Takeda, M. Akiba, T. Yuasa, M Kazam. A. Hoshino. Y. Watanabe. K. Hyodo, F.A Dilmanian, T. Akatsuka and Y Itai, "SPIE-The international Society for Optical Engineering Press" in Physics of Medical Imaging 1996. SPIE Conference Proceedings 2708, Society of Photo-Optical Instrumentation Engineers, Bellingham, WA, 1996, pp. 685-695.

2. T. Takeda, S. Matsushita, J. Wu, Q. Yu, Thet-Thet-Lwin, T. Zenia, T. Yuasa, K Hyodo, F.A. Dilmanian, T. Akatsuka and Y Itai, "World Publishing Corporation International Academic Publishers Press" in Image Visualization and reconstruction edited by X.X. Zheng, et al., IEEE-EMBS Asia-Pacific Conf. on Biomedical Engineering Proceedings 1, 2000, pp 276-277.

3. T. Takeda, A. Momose, Q. Yu, T. Yuasa, F. A. Dilmanian, T. Akatsuka, Y. Itai, Cellular \& Molecular Biology 46, $1077-$ $1088(2000)$.

4. T. Takeda, J. Wu, A. Yoneyama, Y. Tsuchiya, Thet-Thet-Lwin, Y. Hirai, T. Kuroe, T. Yuasa, K. Hyodo, F. A. Dilmanian, T. Akatsuka, "SPIE-The international Society for Optical Engineering Press", in Development in X-ray tomography IV, edited by U. Bonse, SPIE Conference Proceedings 5535, Society of Photo-Optical Instrumentation Engineers, Bellingham, WA, 2004, pp. 380-391.

5. T. Takeda, Nucl. Instrum. Meth. A548, 38-46 (2005)

6. Thet-Thet-Lwin, T. Takeda, J. Wu, Sunaguchi N, Tsuchiya Y, Yuasa T, Dilmanian FA, Minami M, Akatsuka T, 2005, APCMBE Conference Proceedings, Tsukuba, 2005, PA-3-34, pp. 1-4,

7. T. Takeda, A. Momose, Y. Itai, Acad Radiol 2, 799-803 (1995).

8. A. Momose, T. Takeda, Y. Itai, K. Hirano, Nature Med. 2, 473-475 (1996).

9. J. Wu, T. Takeda, Thet Thet Lwin, N. Sunaguchi, T. Yuasa, F. Tadanori, H. Hontani, T. Akatsuka, Med. Imag. Tech. 23, 312-317 (2005)

10. Thet-Thet-Lwin, T. Takeda, J. Wu. Y. Fumikura. K. Iida. S.Kawano, I. Yamaguchi, and Y. Itai, Eur J Nucl Med Mol Imaging 30, 966-973 (2003) 